benefits of IPC and the effects of this on mitochondrial complexity and inner mitochondrial membrane potential $(\psi \mathrm{M})$.

Methods Used Male Sprague Dawley rats $(n=17,322 \pm$ $17 \mathrm{~g}$ ) were heparinized and anesthetized with $80 \mathrm{mg} / \mathrm{kg}$ pentobarbital IP, and their hearts excised and perfused at constant pressure with a non-circulating Langendorff apparatus. Left ventricular (LV) pressure (LVDP) and heart rate (HR) were continually measured with a fluid filled latex balloon attached to a pressure transducer. Treatment groups were: ischemia-reperfusion (IR) $(\mathrm{n}=6)$ : $20 \mathrm{~min}$. perfusion, $30 \mathrm{~min}$. of global ischemia, $45 \mathrm{~min}$. of reperfusion; IPC ( $\mathrm{n}=5)$ : $10 \mathrm{~min}$. perfusion, $3 \mathrm{~min}$. ischemia with $2 \mathrm{~min}$. reperfusion repeated 3 times prior to IR protocol, IPC+ MLA $(n=6): 6$ min. perfusion, 4 min. of infusion of MLA at $233 \mathrm{nM}$, IPC with MLA during reperfusion periods, then IR. Mitochondria were isolated from the LV free wall, stained for $\psi \mathrm{M}$ and for size, and examined by Flow Cytometry with a BD LSRFortessa. Controls (C) $(n=4)$ were freshly excised hearts from similar animals with identical anesthesia.

Summary of Results IPC increased LV work product (LVDP times HR) as a percent of pre-ischemia (\%P) during reperfusion compared to IR control, and this effect was attenuated by MLA pretreatment $(\mathrm{IR}=24.1 \pm 4.5 \% \mathrm{P}$, $\mathrm{IPC}=49.8 \pm 2.8 \% \mathrm{P}, \quad \mathrm{IPC}+\mathrm{MLA}=33.8 \pm 3.5 \% \mathrm{P}, \mathrm{p}<0.01)$. IPC reduced end diastolic pressure from IR levels, and this was partially prevented by MLA treatment $(\mathrm{IR}=78.8$ $\pm 7.7 \mathrm{~mm} \mathrm{Hg}, \quad \mathrm{IPC}=18.8 \pm 6.6 \mathrm{~mm} \mathrm{Hg}, \mathrm{IPC}+\mathrm{MLA}=46.3$ $\pm 8.6 \mathrm{~mm} \mathrm{Hg}, \mathrm{p}<0.05)$. IPC maintained mitochondrial structural complexity compared to IR $(\mathrm{C}=65 \pm 6 \%$ of total mitochondria, $\mathrm{IPC}=61 \pm 5 \%, \mathrm{IR}=32 \pm 4 \%, \mathrm{p}<0.01)$. MLA reduced the effect of IPC on $\psi \mathrm{M}$ in intact mitochondria to IR levels (IR $=67 \pm 10 \%$ of intact population, IPC $=88$ $\pm 3 \%$, IPC + MLA $=71 \pm 4 \%, \mathrm{p}<0.01$ ).

Conclusions Signaling through the a7nAChR is necessary for the effect of IPC on maintaining $\psi \mathrm{M}$ and cardiac contractile function after IR injury.

\title{
Display Posters
}

Wednesday, April 13, 2016

\section{P1 CHOLINERGIC RECEPTOR FUNCTION IN CARDIAC ISCHEMIC PRECONDITIONING}

CW Mullan, ${ }^{1}$ SA Mavropolous, 1,2 K Ojamaa 1,2. 'Hofstra North Shore-LIJ School of Medicine, Raleigh, NY, United States; ${ }^{2}$ The Feinstein Institute for Medical Research, Manhasset, NY, United States

10.1136/jim-2016-000080.41

Purpose of Study Cardiac acetylcholine (ACh) signaling is protective, but the role of $\mathrm{ACh}$ in ischemic preconditioning (IPC) remains largely unknown. We studied the effect of selective alpha-7 nicotinic ACh receptor (a7nAChR) antagonism by methyllycaconitine (MLA) on the functional 